\title{
The approach to the construction of question-answer systems based on the syntagmatic analysis of the text
}

\author{
A A Zarubin ${ }^{1}$, A R Koval ${ }^{1}$, A A Filippov² and V S Moshkin ${ }^{2}$ \\ ${ }^{1}$ The Bonch-Bruevich Saint-Petersburg State University of Telecommunication, Moika street \\ 61, Saint-Petersburg, Russia, 191186 \\ ${ }^{2}$ Ulyanovsk State Technical University, Severny Venetz street 32, Ulyanovsk, Russia, 432027
}

\begin{abstract}
This article describes the application of the mechanisms of syntagmatic patterns in the construction of various types of question-answer (QA) systems and expert systems. QA systems are systems that can take questions and respond to them in a natural language. In most cases, the principles of building question-answer systems are used in the development of decision support systems. The mechanism of syntagmatic patterns is used when processing open-ended questions and when extracting answers to it from semi-structured resources.
\end{abstract}

\section{Introduction}

There is currently a problem of rapid access to information. This problem occurs, for example, when:

- interaction with customers;

- making managerial decisions;

- technical support services, etc

Question-answer systems can be used to solve the problem of operational access to information [1, $2,3,4,5,6]$. Question-answer systems generate a response based on an analysis of the user question. The UIMA architecture is currently used to build question-answer systems [4, 5, 6, 7]. Modern question-answer systems show significant results $[4,5,6]$, but they require the presence of highly skilled specialists for tuning and training $[7,8,9]$.

This article describes an attempt to develop a question-answer system using syntagmatic patterns [10]. Syntagmatic pattern is a template for detecting certain syntagmatic units in the text. Syntagmatic unit is a collection of several words united on the principle of semantic-grammatical-phonetic compatibility. Relations between syntagmatic units are taken into account when using syntagmatic patterns. For example, the syntagmatic pattern "building * the knowledge base" will allow you to find sentences containing syntagmatic units:

- building a knowledge base;

- building a corporate knowledge base;

- building a fuzzy knowledge base, etc.

Our approach is based on the following ideas:

1. The knowledge base of the question-answer system can be generated automatically based on the analysis of unstructured text resources.

2. The use of syntagmatic patterns to organize the structure of the knowledge base of the questionanswer system allows one to effectively search for answers to questions. 


\section{A structure of the $K B$ of the $Q A$ system}

The knowledge base (KB) of our question-answer system has a tree-like structure. Semantic networks are currently actively used in the construction of a KB [8]. Formally, the structure of the KB looks like this:

$$
K B=\langle S P, T D, R\rangle,
$$

where $S P=\left\{S P_{1}, S P_{2}, \ldots, S P_{n}\right\}$ is a set of syntagmatic patterns; $T D=\left\{T D_{1}, T D_{2}, \ldots, T D_{n}\right\}-$ is a set of text data (KB content); $R=\left\{R^{S P}, R^{T D}\right\}$ - is a set of relations of $\mathrm{KB}$ :

$R^{S P}=\left\{R_{1}^{S P}, R_{2}^{S P}, \ldots, R_{n}^{S P}\right\}-$ is a set of relations between the internal nodes of the KB tree;

$R^{T D}=\left\{R_{1}^{T D}, R_{2}^{T D}, \ldots, R_{n}^{T D}\right\}$ - is a set of relations between the internal and terminal nodes of the KB tree.

The internal nodes of the KB tree contain a syntagmatic pattern as a label. Terminal nodes contain text information. The answer to the question will be extracted from this textual information. An example of the KB structure of our question-answer system is shown in Figure 1.

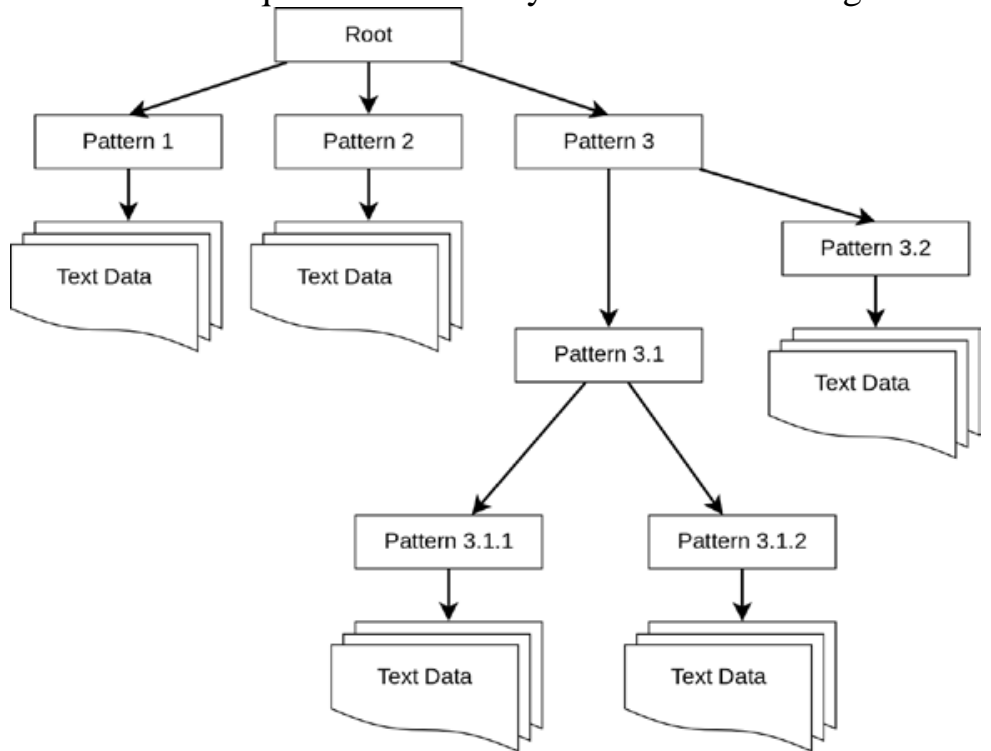

Figure 1. The structure of the knowledge base of our question-answer system.

More general syntagmatic patterns are located closer to the root element of the tree. More precise syntagmatic patterns are located closer to the terminal nodes of the tree. Thus, this knowledge base structure of our question-answer system allows us to find the necessary terminal nodes on the user's question (if the answer to this question is in the knowledge base).

\section{Learning of the $\mathrm{KB}$ of the QA system}

The modified fuzzy C-Means (FCM) fuzzy clustering algorithm is used for learning (building a tree) the KB of our QA system. It is necessary to present each document as an index for the FCM algorithm. Indexing documents consists of the following steps:

1. Download the document.

2. Removing stop words (words that do not have semantic value: prepositions, particles, etc.).

3. Stemming using the Porter algorithm (highlighting the basis of the word).

4. Calculation the frequency of occurrence of words in the document.

The index of the document can be represented as an expression:

$$
I_{d}=\left\{\left(w_{1}^{d}, \mathrm{f}_{\mathrm{i}}^{\mathrm{d}}\right),\left(w_{2}^{d}, \mathrm{f}_{2}^{\mathrm{d}}\right) \ldots,\left(w_{n}^{d}, \mathrm{f}_{\mathrm{n}}^{\mathrm{d}}\right)\right\},
$$

where $w_{i}^{d}-i$-th word of the document $d ; f_{i}^{d}-$ is the frequency of occurrence of $i$-th word in the document $d ; n$-is the number of words in the document $d$.

The modified FCM clustering algorithm is based on minimizing the function: 


$$
F^{F C M}=\sum_{i=1}^{D} \sum_{j=1}^{C} u_{i j}^{m}\left\|I_{i}-I_{j}^{c}\right\|^{2}, 1 \leq m \leq \infty,
$$

where $D-$ is the number of document indexes for clustering; $C$ - is a number of clusters; $m-$ is any real number greater than $1 ; u_{i j}$ - is the degree to which the document index belongs $I_{i}$ to the cluster $j$; $I_{i}-i$-th document index; $I_{j}^{c}-$ is a center of $j$-th cluster; $\left\|I_{i}-I_{j}^{c}\right\|$ - the normalized distance between the index of the document and the center of the cluster.

The FCM algorithm consists of the following steps:

1. Initialization of the matrix of indexes belonging to documents to clusters:

2. Calculation of cluster centers:

$$
U=\left[u_{i j}\right] \text {. }
$$

$$
I_{j}^{c}=\frac{\sum_{i=1}^{D} u_{i j}^{m} \cdot I_{i}}{\sum_{i=1}^{D} u_{i j}^{m}} .
$$

3. Formation of a new membership matrix:

$$
u_{i j}=\frac{1}{\sum_{k=1}^{C}\left(\frac{\left\|I_{i}-I_{j}^{c}\right\|}{\left\|I_{i}-I_{k}^{c}\right\|}\right)^{\frac{2}{m-1}} .}
$$

4. The value of the objective function is calculated. The obtained value is compared with the value at the previous iteration. Clustering is complete if the difference does not exceed the threshold value. Otherwise, go to the second step of the algorithm.

The knowledge base is learned in the process of hierarchical clustering. First, the entire set of document indexes $I^{0}$ is clustered. Clusters are formed after the algorithm is executed. Each cluster obtained contains a subset of the documents of the original set: $I^{1} \cup I^{2} \cup \ldots \cup I^{n}=I^{0}$. A new partition is performed for each cluster received. The split continues as long as the value $\sqrt{D / 2}$ is greater than or equal to 2.

Thus, a tree is constructed whose internal nodes contain indexes of cluster centers, and terminal nodes contain text data. It is necessary to form internal node labels in the form of syntagmatic patterns based on the contents of internal nodes.

\section{An algorithm for constructing syntagmatic patterns}

There are currently many approaches to the analysis of texts in natural language $[1,11,12,13,14,15$, 16, 17]. Statistical and/or linguistic methods for the analysis of texts in natural language underlie such approaches. Methods for the analysis of texts on natural language are also used in the development of QA systems [1, 2, 3, 5, 6, 7].

The analysis of texts on natural language consists of the following steps:

1. Grafematic analysis is the selection of structural elements of the text (sentences, names, dates, etc.).

2. Morphological analysis is the definition of the morphological features of the words of the sentence (part of speech, gender, etc.).

3. Parsing is the selection of the syntactic units of the sentence (subject, predicate, etc.).

4. Semantic analysis is the definition of the meaning of the sentence.

It is sufficient to use the first two steps of the process of text analysis in natural language to form syntagmatic patterns: graphematic and morphological analysis.

The algorithm for syntagmatic patterns is represented in Figure 2.

Thus, internal nodes of the knowledge base tree are marked with syntagmatic patterns as a result of this algorithm. 

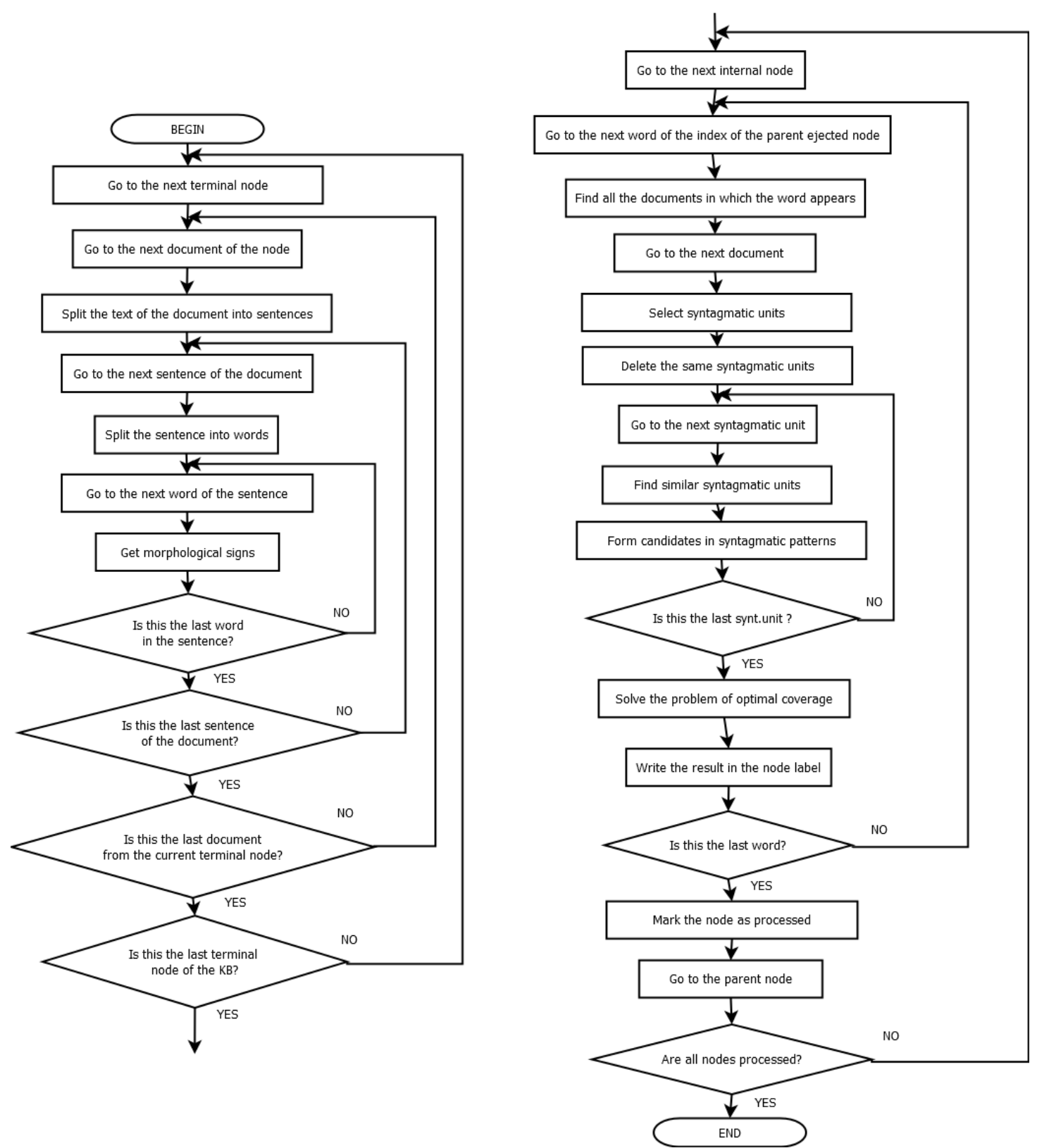

Figure 2. The algorithm for syntagmatic patterns.

\section{The search for the answer to the question in the knowledge base}

The learned KB of our QA system allows us to find answers to the user's requests. First you need to find the required terminal node of the knowledge base. The internal node labels are used to find the most relevant terminal node. Each internal node of the knowledge base is marked with a syntagmatic pattern.

The search algorithm for the most relevant terminal node of the knowledge base tree is represented in Figure 3.

It is necessary to find in the text documents the most relevant sentence after finding the terminal node. The answer to the question is the most relevant sentence.

The search algorithm for the most relevant sentence from the text documents of the terminal node found is represented in Figure 4.

Thus the two algorithms presented above make it possible to organize the search for the most relevant answer to an incoming question. 


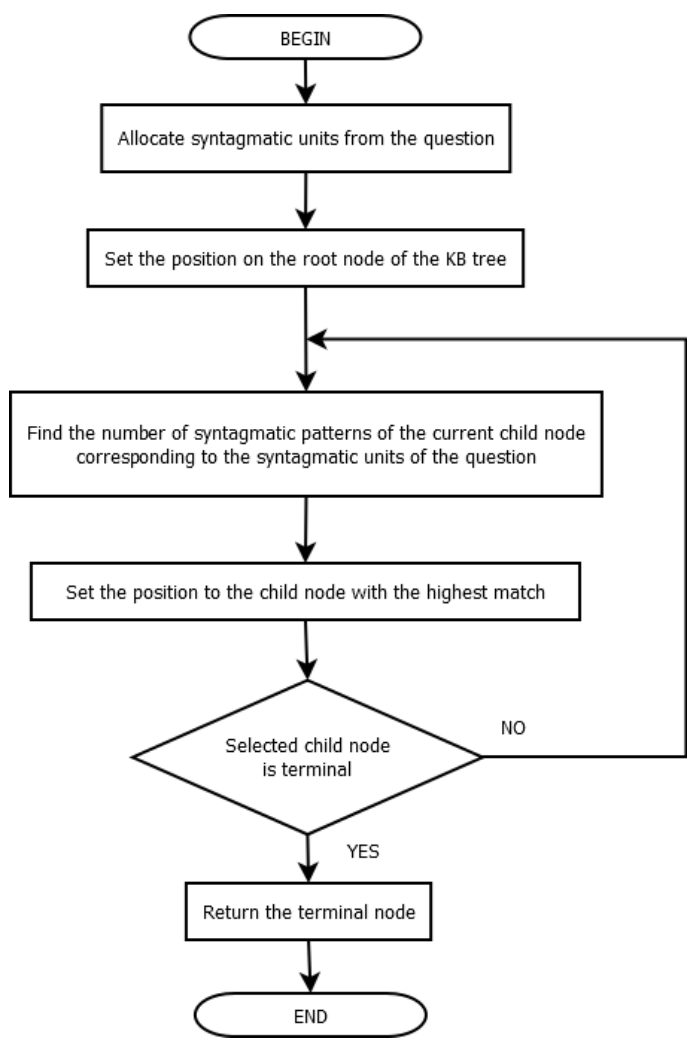

Figure 3. The search algorithm for the most relevant terminal node of the KB tree.

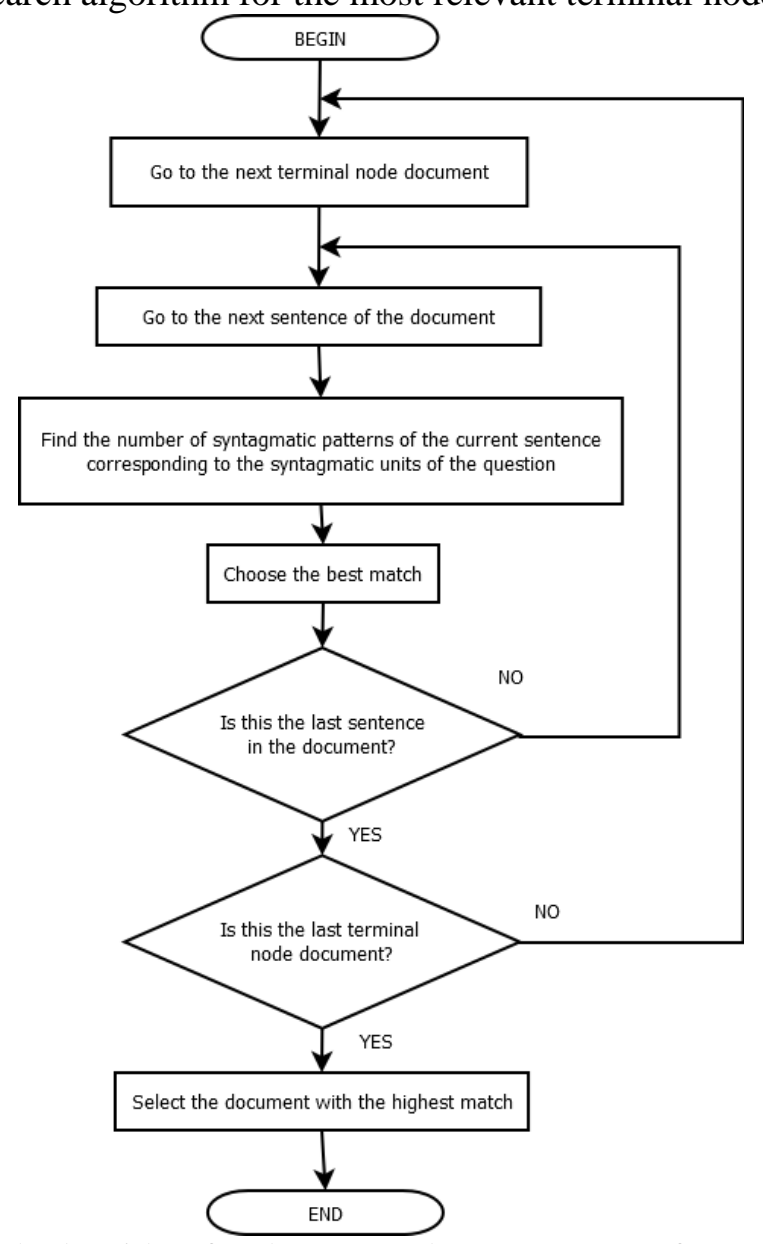

Figure 4. The search algorithm for the most relevant sentence from the text documents. 


\section{Experiments}

The materials of the Sberbank Data Science Contest [18] were used as data for experiments. These materials contain 50,365 entries of the form "paragraph, question, answer." The answer to the question is always the exact text substring of the paragraph, with precision to punctuation and the text register. Each paragraph contains several sentences.

Two question-answer systems were used to conduct the experiment. Their knowledge base was learned using a modified FCM algorithm. The first knowledge base contained many pairs of "termfrequency" as labels of internal nodes. The second knowledge base contained syntagmatic patterns as labels of internal nodes.

A proximity measure was used to find the most relevant document and / or sentence in the first knowledge base. The proximity measure is obtained using the square of the Euclidean distance:

$$
\operatorname{Dist}\left(I_{i}^{c}, I^{q}\right)=\sum_{w=1}^{W}\left(f_{w}^{c}-f_{w}^{q}\right)^{2},
$$

where $I_{i}^{c}-$ is the index of the $i$-th terminal node document $c$; $I^{q}-$ is the index of the received question; $W-$ is the number of words in the index $I_{i}^{c} ; f_{w}^{c}, f_{w}^{q}-$ is the frequency of occurrence of the word $w$ in the indexes $I_{i}^{c}$ and $I^{q}$.

The most relevant document and / or sentence is a document and / or sentence with a minimum proximity measure.

During the experiment 50,365 questions were submitted to both question-answer systems. The sentence from the paragraph was given as an answer to the question. The result was considered successful if the reference answer was a substring of the found sentence.

The results of the experiments are presented in Table 1.

Table 1. The comparison of statistical and syntagmatic approaches to the implementation of the question-answer system.

\begin{tabular}{lrr}
\hline Type of KB & Number of errors & Percent of errors \\
\hline Vector & 23178 & 46,0 \\
Syntagmatic & 9723 & 19,3 \\
\hline
\end{tabular}

As can be seen from the results of the experiments, the syntagmatic approach to the implementation of the question-answer system made it possible to reduce the number of errors from $46 \%$ to $19.3 \%$.

\section{Conclusion}

Thus, the developed syntagmatic approach to the development of question-answer systems is effective. This approach can be used to develop the following types of software systems:

- the system for automating the process of interaction with customers based on the analysis of the knowledge base and corporate correspondence;

- decision support system based on the analysis of the knowledge base and use cases;

- the system of verification of information flows of the enterprise to ensure information security;

- the system for automating the work of the technical support service based on the analysis of the knowledge base and use cases.

In the future, we plan to modify the developed approach by finding answers to questions in an implicit form.

\section{References}

[1] Jurafsky D and Martin J H 2018 Speech and Language Processing (Access mode: https://web.stanford.edu/ jurafsky/slp3/28.pdf) (03.05.2018)

[2] Berant J, Chou A, Frostig R and Liang P 2013 Semantic parsing on freebase from questionanswer pairs Proceedings of the Conference on Empirical Methods in Natural Language Processing (EMNLP) 1533-1544 
[3] Bordes A, Chopra S and Weston J 2018 Question answering with subgraph embeddings (Access mode: https://arxiv.org/pdf/1406.3676.pdf) (03.05.2018)

[4] Epstein E A, Schor M I, Iyer B, Lally A, Brown E W and Cwiklik J 2012 Making watson fast IBM Journal of Research and Development 56(3.4) 15-19

[5] Ferrucci D, Brown E, Chu-Carroll J, Fan J, Gondek D, Kalyanpur A A, Lally A, Murdock J W, Nyberg E and Prager J 2010 Building watson: An overview of the deepqa project AI magazine 31(3) 59-79

[6] Gallagher S, Zadrozhny W, Shalaby W and Avadhani A 2018 Watsonsim: Overview of a question answering engine (Access mode: https://arxiv.org/pdf/1412.0879.pdf) (03.05.2018)

[7] Ferrucci D and Lally A 2004 UIMA: An architectural approach to unstructured information processing in the corporate research environment Nat. Lang. Eng 10(3-4) 327-348

[8] Bollacker K, Evans C, Paritosh P, Sturge T and Taylor J 2008 Freebase: a collaboratively created graph database for structuring human knowledge Proceedings of the ACM SIGMOD International Conference on Management of Data 1247-1250

[9] Chen D and Manning C D 2014 A fast and accurate dependency parser using neural networks Proceedings of the Conference on Empirical Methods in Natural Language Processing (EMNLP) 740-750

[10] Zarubin A, Koval A, Filippov A and Moshkin V 2017 Application of syntagmatic patterns to evaluate answers to open-ended questions Proceedings of the Communications in Computer and Information Science (CITDS) 150-162

[11] Zarubin A A, Koval A R, Moshkin V S and Filippov A A 2017 Construction of the problem area ontology based on the syntagmatic analysis of external wiki-resources CEUR Workshop Proceeding 1903 128-134

[12] Boyarskiy K K, Kanevskiy Ye A 2015 Semantic and syntactic parser SemSin Scientific and Technical Herald of Information Technologies, Mechanics and Optics 5 869-876

[13] Artemov M A, Vladimirov A N and Seleznev K E 2013 Review of Russian NLP systems (Access mode: http://www.vestnik.vsu.ru/pdf/analiz/2013/02/2013-02-31.pdf) (03.05.2018)

[14] Automatic text processing (Access mode: http://aot.ru) (03.05.2018)

[15] Lally A, Prager J M, McCord M C, Boguraev B, Patwardhan S, Fan J, Fodor P and Chu-Carroll J 2012 Question analysis: How watson reads a clue IBM Journal of Research and Development 56(3.4) 2-14

[16] Mikhaylov D V, Kozlov A P and Emelyanov G M 2017 An approach based on analysis of ngrams on links of words to extract the knowledge and relevant linguistic means on subjectoriented text sets Computer Optics 41(3) 461-471 DOI: 10.18287/2412-6179-2017-41-3-461471

[17] Mikhaylov D V, Kozlov A P and Emelyanov G M 2016 Extraction the knowledge and relevant linguistic means with efficiency estimation for formation of subject-oriented text sets Computer Optics 40(4) 572-582 DOI: 10.18287/2412-6179-2016-40-4-572-582

[18] Sberbank Data Science Contest (Access mode: https://contest.sdsj.ru/) (03.05.2018)

\section{Acknowledgments}

This paper has been approved within the framework of the federal target project " $R \& D$ for Priority Areas of the Russian Science-and-Technology Complex Development for 2014-2020", government contract No 14.607.21.0164 on the subject "The development of architecture, methods and models to build software and hardware complex semantic analysis of semi-structured information resources on the Russian element base” (Application Code 2016-14-579-0009-0687). 